\title{
Article \\ Fibroblast Growth Factor 19 and Fibroblast Growth Factor 21 Regulation in Obese Diabetics, and Non-Alcoholic Fatty Liver Disease after Gastric Bypass
}

\author{
Jiun-Yu Guo ${ }^{1}$ (D), Hsin-Hung Chen ${ }^{2}$, Wei-Jei Lee ${ }^{3}$, Shu-Chun Chen ${ }^{4}$, Shou-Dong Lee ${ }^{5}$ and Chih-Yen Chen ${ }^{6,7,8,9, *}$ \\ 1 Division of Cardiology, Department of Medicine, Taipei Veterans General Hospital, Taipei 112201, Taiwan; \\ jyguo@vghtpe.gov.tw \\ 2 Department of Nutrition and Health Sciences, Chang Jung Christian University, Tainan 71101, Taiwan; \\ hsinhung@mail.cjcu.edu.tw \\ 3 Department of Surgery, Min-Sheng General Hospital, Taoyuan 330056, Taiwan; \\ wjlee_obessurg_tw@yahoo.com.tw \\ 4 Department of Nursing, Chang-Gung Institute of Technology, Taoyuan 33303, Taiwan; N002916@e-ms.com.tw \\ 5 Division of Gastroenterology, Department of Internal Medicine, Cheng-Hsin General Hospital, \\ Taipei 11220, Taiwan; ch9318@chgh.org.tw \\ 6 Division of Gastroenterology and Hepatology, Department of Medicine, Taipei Veterans General Hospital, \\ Taipei 112201, Taiwan \\ 7 Faculty of Medicine and Institute of Emergency and Critical Medicine, College of Medicine, \\ National Yang Ming Chiao Tung University, Taipei 11221, Taiwan \\ 8 Chinese Taipei Society for the Study of Obesity, Taipei 110301, Taiwan \\ 9 Taiwan Association for the Study of Small Intestinal Diseases, Taoyuan 333423, Taiwan \\ * Correspondence: chency@vghtpe.gov.tw; Tel.: +886-2-28712121 (ext. 2050); Fax: +886-2-28711058
}

check for updates

Citation: Guo, J.-Y.; Chen, H.-H.; Lee, W.-J.; Chen, S.-C.; Lee, S.-D.; Chen, C.-Y. Fibroblast Growth Factor 19 and Fibroblast Growth Factor 21 Regulation in Obese Diabetics, and Non-Alcoholic Fatty Liver Disease after Gastric Bypass. Nutrients 2022, 14, 645. https://doi.org/10.3390/ nu14030645

Academic Editor: Toshihiko Yada

Received: 29 December 2021

Accepted: 29 January 2022

Published: 2 February 2022

Publisher's Note: MDPI stays neutral with regard to jurisdictional claims in published maps and institutional affiliations.

Copyright: (c) 2022 by the authors. Licensee MDPI, Basel, Switzerland. This article is an open access article distributed under the terms and conditions of the Creative Commons Attribution (CC BY) license (https:/ / creativecommons.org/licenses/by/ $4.0 /)$.

\begin{abstract}
Background: Gastric bypass (GB) is an effective treatment for those who are morbidly obese with coexisting type 2 diabetes mellitus (T2DM) or non-alcoholic fatty liver disease (NAFLD). Fibroblast growth factors (FGFs) are involved in the regulation of energy metabolism. Methods: We investigated the roles of FGF 19, FGF 21, and total bile acid among those with morbidly obese and T2DM undergoing GB. A total of 35 patients were enrolled. Plasma FGF 19, FGF 21, and total bile acid levels were measured before surgery (M0), 3 months (M3), and 12 months (M12) after surgery, while the hepatic steatosis index (HSI) was calculated before and after surgery. Results: Obese patients with T2DM after GB presented with increased serum FGF 19 levels $(p=0.024)$ and decreased total bile acid $(p=0.01)$ and FGF 21 levels $(p=0.005)$. DM complete remitters had a higher FGF 19 level at M3 ( $p=0.004)$ compared with DM non-complete remitters. Fatty liver improvers tended to have lower FGF 21 ( $p=0.05)$ compared with non-improvers at M12. Conclusion: Changes in FGF 19 and FGF 21 play differential roles in DM remission and NAFLD improvement for patients after GB. Early increases in serum FGF 19 levels may predict complete remission of T2DM, while a decline in serum FGF 21 levels may reflect the improvement of NAFLD after GB.
\end{abstract}

Keywords: obesity; diabetes mellitus; FGF 19; FGF 21; total bile acid; non-alcoholic fatty liver disease; gastric bypass

\section{Introduction}

Obesity has been a global concern for the past 50 years and the prevalence has increased significantly over the past decade [1]. Obesity represents a major health challenge because it substantially increases the risk of metabolic diseases, including type 2 diabetes mellitus (T2DM) and non-alcoholic fatty liver disease (NAFLD) [1,2].

Body weight reduction is an important approach in reducing insulin resistance and improving NAFLD. Weight loss has been shown as one of the strongest predictors of improved insulin sensitivity [3]. The magnitude of weight loss is also correlated with the improvement of NAFLD [4]. 
Surgical intervention is considered an important approach, especially for morbidly obese patients with T2DM, medically resistant arterial hypertension, or comorbidities that are expected to improve with weight loss [5]. Gastric bypass, a widely adapted surgical technique, is one of the most effective methods to combat obesity and remit T2DM [6]. Nevertheless, there are many patients whose DM and NAFLD fail to improve despite receiving these interventions [7]. Furthermore, the mechanisms by which it causes weight loss and T2DM and NAFLD resolutions are not well elucidated [8].

Numerous studies have attempted to identify robust biological and clinical predictors of DM remission after bariatric surgery $[9,10]$. On the other hand, studies for improving NAFLD are relatively lacking and mostly limited to animal studies [11]. More biomarkers from the blood as surrogates in the evaluation of NAFLD to replace paired liver biopsy and reduce the suffering of the patients are desperately needed [12].

The human fibroblast growth factor (FGF) family contains at least 22 members involving the biological processes of cell growth, differentiation, development, and metabolism [13]. Aside from most FGFs presenting functions as autocrine or paracrine factors, FGF 19, FGF 21, and FGF 23 lack the conventional FGF heparin-binding domain and possess the ability to elicit endocrine actions, functioning as hormones [13]. Emerging evidence demonstrates the potential role of the FGF family in energy metabolism and in counteracting obesity, especially FGF 19 and FGF 21 [14]. Animal studies have shown that overexpression of FGF 19 or FGF 21 or treatment with recombinant protein enhanced metabolic rates and decreased fat mass, in addition to demonstrating improvements in glucose metabolism, insulin sensitivity, and lipid profiles [15-18].

Bile acids have a significant relationship with energy balance. Farnesoid-X-receptor (FXR) regulates bile acid homeostasis by regulating the transcription of several enterohepatic genes. The activation of the transcription factor FXR by bile acids provokes the subsequent secretion of FGF 19 [19]. Human FGF 19 is expressed in the ileal enterocytes of the small intestine. FGF 19, secreted into the portal circulation, has a pronounced diurnal rhythm, with peaks occurring 90-120 min after serum bile acid levels after food intake. $\beta$-Klotho (KLB) works as a co-receptor and supports endocrine signaling via binding with FGF receptor (FGFr) 4 [20]. The binding of FGF 19 to the hepatocyte cell surface FGFr 4/KLB complex leads to negative feedback and reduces hepatic bile salt synthesis [21]. Mice with impaired function for gut secretion of FGF 19 show significantly impaired weight loss and glucose improvement following bariatric surgery [15]. Collective data also reveal that the serum FGF 19 levels are decreased in patients with T2DM [22].

Apart from FGF 19, FGF 21 is expressed in multiple tissues, including the liver, brown adipose tissue, white adipose tissue, and pancreas [23]. Under normal physiologic status, most circulating FGF 21 originates from the liver [24]. Secretion of FGF 21 is provoked significantly by excess food intake, ketogenic, high-carbohydrate diets, or protein restriction [25]. The expression of the FGF 21 gene depends on several pathways. Increased circulating free fatty acids and prolonged fasting promote the transcriptional activation of FGF 21 by the peroxisome proliferator-activated receptor $\alpha$-mediated pathway [26,27]. A high-glucose diet activates carbohydrate-response element-binding protein (ChREBP) and enhances FGF 21 secretion [28]. Furthermore, general control nonderepressible 2 (GCN2) would be activated when encountering amino acid deficiency, leading to FGF 21 transcription [29]. Metabolic stresses such as obesity, T2DM, or NAFLD are also responsible for inducing the expression and/or signaling of FGF 21 [30]. The FGF 21-dependent signaling of downstream FGFr is extremely complicated and well-debated [31]. Based on evidence from a recent study, FGF 21 stimulates hepatic fatty acid oxidation, ketogenesis, and gluconeogenesis, and suppresses lipogenesis [25]. FGF 21 reduced plasma glucose and triglycerides to a nearly normal level in an animal model [32].

Although recent studies provide clues regarding the dynamics of FGF 19 and FGF 21 in patients receiving bariatric surgery $[8,33]$, the information is limited to sleeve gastrectomy [8]. Moreover, different characteristics between those with and without the improvement of obesity-related comorbidities were also lacking [33]. 
The main purpose of our study was to evaluate the effect of GB on changes in serum FGF 19 and FGF 21 levels. Furthermore, we also determined the relationship between both blood biomarkers and the improvement of either T2DM or NAFLD.

\section{Materials and Methods}

\subsection{Study Design and Patients}

This was a hospital-based prospective observational study. Obese patients with T2DM receiving GB surgery were enrolled in the study. The study was conducted in accordance with the Declaration of Helsinki and the study protocol was approved by the institutional review board (approval number: MSIRB2015017, YM 103127E, 201002037IC, 2011-09-015IC, CHGH-IRB (405)102A-52)).

The inclusion criteria were as follows: (1) been diagnosed with T2DM for more than 6 months with a baseline hemoglobin $\mathrm{A} 1 \mathrm{c}(\mathrm{HbA} 1 \mathrm{c})$ level $>8 \%$; (2) receiving regular medical treatment for T2DM, including therapeutic nutritional therapy, oral anti-diabetic agents, or insulin under the evaluation of specialists; (2) body mass index (BMI) $\geq 32.5 \mathrm{~kg} / \mathrm{m}^{2}$; (3) willingness to receive additional treatment, including diet control and lifestyle modifications; (4) willingness to accept follow-up appointment, and provided the informed consent documents.

We excluded patients who had the following diagnosis or baseline characteristics: (1) cancer within the previous 5 years; (2) human immunodeficiency virus infection or active pulmonary tuberculosis; (3) unstable cardiovascular condition within the past 6 months; (4) pulmonary embolisms; (5) serum creatinine levels $>2.0 \mathrm{mg} / \mathrm{dL}$; (6) chronic hepatitis B or C, liver cirrhosis, inflammatory bowel disease, or acromegaly; (7) history of organ transplantation or other bariatric surgery; (8) alcohol use disorders or substance use disorder; or (9) those who were uncooperative.

All study patients returned for a monthly follow-up appointment after surgery. The patients received a face-to-face consultation with dietitians and reported a food diary log. Clinical anthropometry and laboratory assessments were performed simultaneously.

\subsection{Surgical Technique of Gastric Bypass (GB)}

GB was performed as described in our previous studies [34-36]. A five-port technique was used. The dissection begins directly on the lesser curvature, and a 15- to 20-mL gastric pouch is created using multiple EndoGIA-45 staplers (US Surgical Corp). Patients are then placed in a neutral position for the creation of the jejunostomy. The jejunum is divided $50 \mathrm{~cm}$ distal to the ligament of Treitz. A stapled end-to-side jejunojejunostomy is performed with a $100 \mathrm{~cm}$ Roux limb. The enteroenterostomy defect and all mesenteric defects are closed with continuous sutures. The Roux limb is tunneled via a retrocolic, retrogastric path and positioned near the transected gastric pouch. The CEEA-21 anvil (US Surgical Corp) is pulled into the gastric pouch transorally following the previous study [37]. The CEEA stapler is then inserted through the Roux limb to perform the end-to-side gastrojejunostomy. After the test for air leak, the trocar fascial defects are closed. Two hemovac drains are left in the lesser sac and left subphrenic space separately [38].

\subsection{Metabolic Profiles and Blood Sampling}

All study subjects received clinical anthropometry and laboratory assessments on the day before surgery as baseline (M0) and at 3 months (M3) and 12 months (M12) after surgery. Metabolic profiles included anthropometry measurements (body weight, waist circumference, body mass index (BMI), and a body shape index (ABSI)), and systolic and diastolic blood pressure. The patients were required to fast overnight before each blood sample collection. Blood samples were obtained from the median cubital vein between 8 and 11 o'clock in the morning and immediately transferred into a chilled glass tube containing disodium EDTA $(1 \mathrm{mg} / \mathrm{mL})$ and aprotinin (500 units $/ \mathrm{mL})$. The samples were stored on ice during collection. They were further centrifuged, plasma-separated, aliquoted into polypropylene tubes, and stored at $-20{ }^{\circ} \mathrm{C}$ before receiving analysis. 
Laboratory assessments included serum levels of total cholesterol, triglycerides, highdensity lipoprotein cholesterol (HDL-C), low-density lipoprotein cholesterol (LDL-C), fasting blood sugar, hemoglobin A1c (HbA1c), c-peptide, insulin, creatinine, uric acid, and liver function test (alanine transaminase (ALT), aspartate transaminase (AST), alkaline phosphatase (Alk-p), and gamma-glutamyl transferase $(\gamma-G T))$. All data are reported as means \pm standard deviations.

\subsection{Measurement of the Plasma FGF 19, FGF 21, and Serum Total Bile Acid Levels}

The fasting blood samples obtained were used to determine the plasma levels of FGF 19 and FGF 21, and serum levels of total bile acids. Enzyme immunoassays for plasma FGF 19 and FGF 21 (R\&D Systems, Minneapolis, MN, USA) were performed. Fasting total serum bile acids were assayed using the $3 \alpha$-hydroxysteroid dehydrogenase method (Fumouze Diagnostics, Levallois-Perret, France). All measurements were performed in duplicate.

\subsection{Definition of DM Complete Remission and Insulin Resistance}

In our study, DM complete remission is defined as a return to normal measures of glucose metabolism with $\mathrm{HbA} 1 \mathrm{c}$ in the normal range $(<6.0 \%(42 \mathrm{mmol} / \mathrm{mol}))$, which was adopted in most previous investigations [39]. We define $\mathrm{HbA} 1 \mathrm{c}<6.0 \%$ after 12 months after GB as DM complete remitters.

Insulin resistance was evaluated by using the homeostasis model assessment of insulin resistance $(\mathrm{HOMA}-\mathrm{IR})$, defined as fasting plasma glucose level $(\mathrm{mmol} / \mathrm{L}) \times$ fasting immunoreactive insulin level $(\mu \mathrm{U} / \mathrm{mL}) / 22.5$. The $\beta$-cell function was assessed using the homeostatic model assessment of $\beta$-cell function (HOMA- $\beta$ ), with the formula of [20 $\times$ fasting immunoreactive insulin level $(\mu \mathrm{U} / \mathrm{mL})] /[$ fasting plasma glucose level $(\mathrm{mmol} / \mathrm{L})-3.5$ ] [40]. The variation of the area under the curve of plasma FGF 19 levels per minute ( $\triangle$ AUC of plasma amylin) during the OGTT was calculated with the trapezoidal method [41].

\subsection{Definition of NAFLD Based on the Hepatic Steatosis Index (HSI)}

NAFLD has been considered as a continuum from obesity to metabolic syndrome and diabetes [42]. The gold standard to evaluate the magnitude of NAFLD depends on paired liver biopsy. However, liver biopsy may lead to patient discomfort and several complications, including bleeding, organ trauma, and even patient mortality [43]. Therefore, this approach is less feasible in clinical practice due to patient safety and a high patient refusal rate $[12,44]$. The hepatic steatosis index (HSI) was adapted for the clinical assessment of NAFLD and has been validated with imaging, including ultrasonography and magnetic resonance imaging [45]. HSI was defined as $8 \times($ ALT $/$ AST ratio $)+$ BMI (+2, if female; +2, if diabetes mellitus). A cut-off value of HSI $>36.0$ may determine NAFLD with a specificity of $92.4 \%$ [46]. Based on this concept, our study defines HSI $<36.0$ at 12 months after GB as HSI improvers (HSI-I).

\subsection{Statistical Analysis}

SAS (version 9.4) was applied for the data analyses. The paired $t$-test was used to compare preoperative age, BMI, waist circumference, total body fat percentage, and resting metabolic rate among patients who underwent GB between the preoperative period and 3 and 12 months after surgery.

Postoperative serum biochemical data (except BMI) were analyzed using an ANCOVA model to adjust for preoperative age and gender. The paired Student $t$-test was used in comparisons between preoperative data with data at each time point at 3 or 12 months postoperatively.

A trend analysis (to obtain $p$ values for the trend) was performed using the repeated GLM to explore whether GB had a significantly different effect on postoperative indicators within 3 or 12 months postoperatively. 
The correlations among different postoperative indicators to explain possible physiological pathways were evaluated using the Pearson correlation. A $p$-value of $<0.05$ was considered statistically significant.

\section{Results}

\subsection{Changes in Metabolic Profiles and Laboratory Data after GB}

A total of 35 obese patients (12 males and 23 females) with T2DM who underwent GB were enrolled. The baseline average age, body weight, and BMI were $44.8 \pm 9.7$ years old, $84.8 \pm 14.1 \mathrm{~kg}$, and $31.6 \pm 4.6 \mathrm{~kg} / \mathrm{m}^{2}$, respectively. The duration of T2DM was $5.8 \pm 4.9$ years. All enrolled patients received GB and were followed up for more than 1 year subsequently.

Changes in metabolic profiles and laboratory data are reported in Table 1. Body weight, BMI, waist circumference, and ABSI showed significant improvements 1 year after GB $(p<0.001)$. Diabetes-related parameters, including fasting blood glucose, $\mathrm{HbA1c}$, c-peptide, and insulin level, also showed a significant decline $(p<0.05)$. Liver function tests, including ALT, AST, and Alk-p levels, showed no significant change; however, a significant decline in $\gamma$-GT level was observed $(p=0.006)$. As for the lipid profile, HDL-C increased, and triglycerides decreased $(p<0.05)$, while total cholesterol and LDL levels did not change significantly. Decreases in uric acid levels were also demonstrated $(p=0.019)$.

Table 1. Characteristics of the obese patients with type 2 diabetes mellitus before gastric bypass (M0) and 3 months (M3) and 1 year (M12) postoperatively.

\begin{tabular}{|c|c|c|c|c|}
\hline$n=35$ & M0 & M3 & M12 & $p$ Value \\
\hline \multicolumn{5}{|l|}{ Metabolic profile } \\
\hline Body weight (kg) & $84.78 \pm 14.12$ & $69.64 \pm 9.68$ & $63.86 \pm 6.75$ & $<0.001$ \\
\hline BMI $\left(\mathrm{kg} / \mathrm{m}^{2}\right)$ & $31.63 \pm 4.62$ & $26.14 \pm 3.19$ & $24.41 \pm 2.58$ & $<0.001$ \\
\hline Waist circumference $(\mathrm{cm})$ & $103.60 \pm 10.25$ & $90.40 \pm 7.96$ & $82.33 \pm 5.30$ & $<0.001$ \\
\hline Excess weight loss (\%) & & $19.00 \pm 22.99$ & $18.43 \pm 10.83$ & 0.346 \\
\hline ABSI & $0.081 \pm 0.005$ & $0.077 \pm 0.017$ & $0.077 \pm 0.004$ & $<0.001$ \\
\hline Systolic blood pressure (mmHg) & $136.09 \pm 14.84$ & $132.54 \pm 21.34$ & $126.86 \pm 16.31$ & 0.076 \\
\hline $\begin{array}{l}\text { Diastolic blood pressure }(\mathrm{mmHg}) \\
\text { Laboratory data }\end{array}$ & $85.54 \pm 10.73$ & $81.23 \pm 16.30$ & $79.67 \pm 14.15$ & 0.277 \\
\hline Creatinine $(\mathrm{mg} / \mathrm{dL})$ & $0.79 \pm 0.32$ & $0.76 \pm 0.28$ & $0.71 \pm 0.29$ & $<0.001$ \\
\hline Fasting blood glucose (mg/dL) & $176.66 \pm 70.64$ & $127.03 \pm 46.69$ & $114.09 \pm 31.11$ & $<0.001$ \\
\hline $\operatorname{HbA} 1 \mathrm{c}(\%)$ & $9.29 \pm 1.52$ & $7.07 \pm 1.62$ & $6.50 \pm 1.16$ & $<0.001$ \\
\hline C-peptide (mg/dL) & $2.65 \pm 1.19$ & $1.69 \pm 0.59$ & $1.39 \pm 0.50$ & $<0.001$ \\
\hline Insulin (mU/L) & $23.16 \pm 28.90$ & $7.10 \pm 6.89$ & $5.83 \pm 6.86$ & 0.007 \\
\hline HOMA-IR index & $9.91 \pm 13.39$ & $2.03 \pm 1.99$ & $1.65 \pm 2.02$ & $<0.001$ \\
\hline HOMA- $\beta$ index & $1.15 \pm 1.95$ & $0.54 \pm 0.59$ & $0.50 \pm 0.67$ & 0.060 \\
\hline $\operatorname{ALT}(\mathrm{U} / \mathrm{L})$ & $41.60 \pm 33.46$ & $35.43 \pm 33.42$ & $33.29 \pm 33.20$ & 0.251 \\
\hline AST (U/L) & $32.17 \pm 28.09$ & $29.61 \pm 20.95$ & $28.90 \pm 26.14$ & 0.364 \\
\hline Alk-p (U/L) & $61.46 \pm 19.09$ & $81.04 \pm 40.46$ & $69.81 \pm 19.46$ & 0.268 \\
\hline$\gamma-\mathrm{GT}(\mathrm{U} / \mathrm{L})$ & $42.29 \pm 27.87$ & $33.37 \pm 42.45$ & $25.10 \pm 22.18$ & 0.006 \\
\hline Total cholesterol (mg/dL) & $193.60 \pm 42.93$ & $176.21 \pm 34.03$ & $168.09 \pm 33.23$ & 0.063 \\
\hline Triglyceride (mg/dL) & $231.37 \pm 217.51$ & $127.36 \pm 57.10$ & $105.25 \pm 45.93$ & 0.006 \\
\hline HDL-C (mg/dL) & $41.57 \pm 7.80$ & $37.93 \pm 7.83$ & $46.31 \pm 10.23$ & $<0.001$ \\
\hline LDL-C (mg/dL) & $117.89 \pm 33.89$ & $117.14 \pm 31.26$ & $105.69 \pm 30.31$ & 0.254 \\
\hline Uric acid (mg/dL) & $5.64 \pm 1.63$ & $5.43 \pm 1.46$ & $5.11 \pm 1.50$ & 0.019 \\
\hline Total bile acid $(\mu \mathrm{M})$ & $10.07 \pm 4.33$ & $11.78 \pm 9.32$ & $8.31 \pm 4.95$ & 0.010 \\
\hline FGF $19(\mathrm{pg} / \mathrm{mL})$ & $84.20 \pm 61.31$ & $141.76 \pm 108.70$ & $142.69 \pm 100.21$ & 0.024 \\
\hline FGF $21(\mathrm{pg} / \mathrm{mL})$ & $320.06 \pm 238.96$ & $416.99 \pm 375.86$ & $230.24 \pm 123.71$ & 0.005 \\
\hline HSI & $45.89 \pm 6.39$ & $38.96 \pm 4.16$ & $36.25 \pm 2.61$ & $<0.001$ \\
\hline
\end{tabular}

ABSI, a body shape index; BMI, body mass index; HbA1c, hemoglobin A1c; ALT, alanine aminotransferase; AST, aspartate transaminase; Alk-p, alkaline phosphatase; $\gamma$-GT, gamma-glutamyl transferase; HDL-C, highdensity lipoprotein cholesterol; HOMA, homeostasis model assessment; IR, insulin resistance; LDL-C, low-density lipoprotein cholesterol; FGF, fibroblast growth factor; HSI, hepatic steatosis index. 
Variations in serum levels among FGF 19, FGF 21, and total bile acids before the operation and after 3 months and 12 months are presented in Figure 1. All three markers showed a significant trend of change after GB ( $p=0.024,0.005$, and 0.010 , respectively). The total bile acid level changed from $10.07 \pm 4.33 \mu \mathrm{M}$ at $\mathrm{M} 0$ to $11.78 \pm 9.32 \mu \mathrm{M}$ at $\mathrm{M} 3$ and to $8.31 \pm 4.95 \mu \mathrm{M}$ at M12 ( $p=0.023$ between M3 and M12). The FGF 19 level increased from $84.20 \pm 61.31 \mathrm{pg} / \mathrm{mL}$ at M0 to $141.76 \pm 108.70 \mathrm{pg} / \mathrm{mL}$ at M3 and to $142.69 \pm 100.21 \mathrm{pg} / \mathrm{mL}$ at M12 ( $p=0.016$ between M0 and M3, $p=0.002$ between M0 and M12). The FGF 21 level changed from $320.06 \pm 238.96 \mathrm{pg} / \mathrm{mL}$ at M0 to $416.99 \pm 375.86 \mathrm{pg} / \mathrm{mL}$ at M3 and to $230.24 \pm 123.71 \mathrm{pg} / \mathrm{mL}$ at M12 ( $p=0.049$ between M0 and M3, $p=0.005$ between M0 and M12).

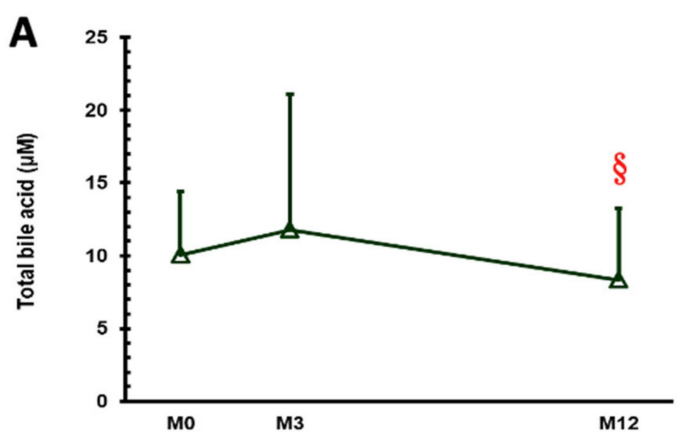

B

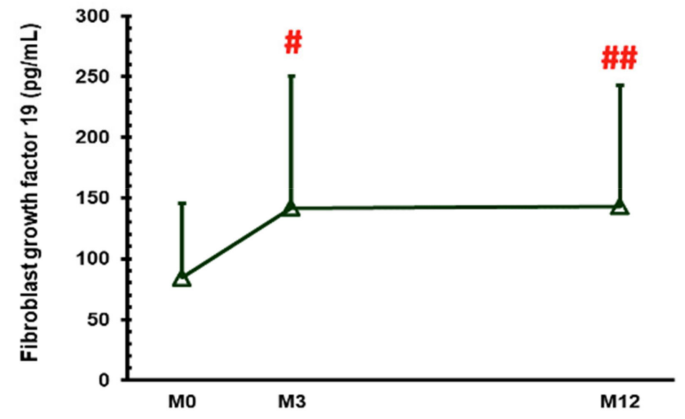

C

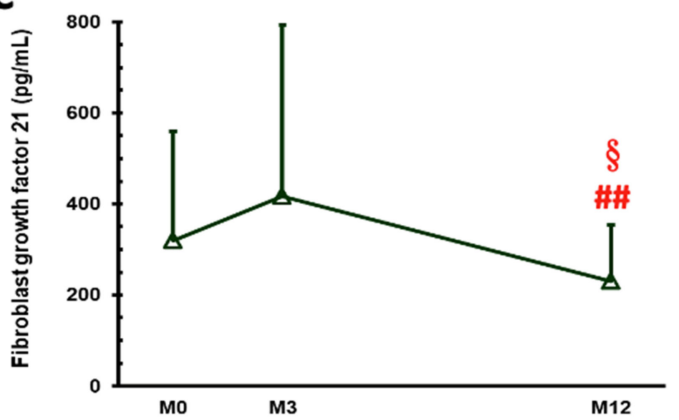

Figure 1. Serum levels of total bile acids (A), fibroblast growth factor 19 (B), and fibroblast growth factor 21 (C) in patients with obesity and type 2 diabetes before gastric bypass (M0) and 3 months (M3) and 1 year (M12) (B) after surgery. \# $p<0.05$, \#\# $p<0.01$ compared with M0, and $\S p<0.05$ compared with M3.

Serum levels of FGF 19 and FGF 21 for subjects during the three periods were intergraded and the correlations with indicators of diabetes and NAFLD are shown in Figure 2. FGF 19 had a significant negative correlation with serum c-peptide $(\mathrm{r}=-0.286, p=0.006$, Figure 2A) and HbA1c level ( $\mathrm{r}=-0.308, p=0.003$, Figure 2B). On the other hand, FGF 21 had a significant positive correlation with serum HbA1c $(r=0.209, p=0.047$, Figure 2C) and total bile acids $(r=0.273, p=0.005$, Figure $2 \mathrm{D})$, respectively. 
A

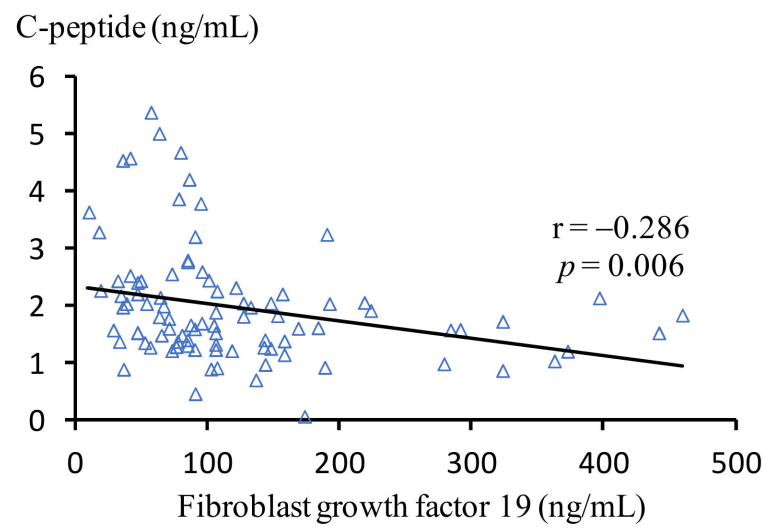

$\mathrm{C}$

C-peptide $(\mathrm{ng} / \mathrm{mL})$

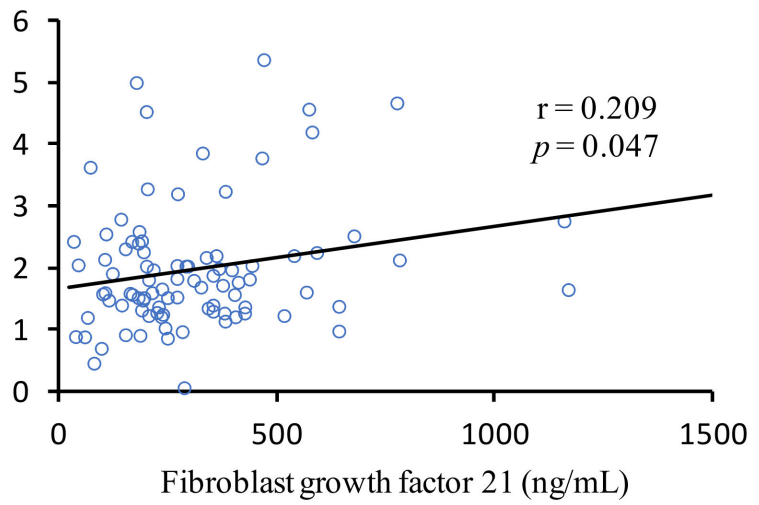

B

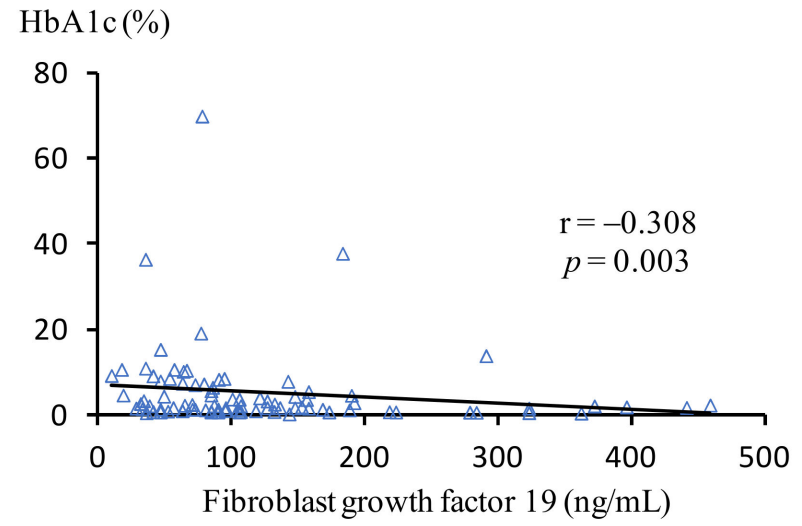

$\mathrm{D}$

Total bile acid $(\mu \mathrm{M})$

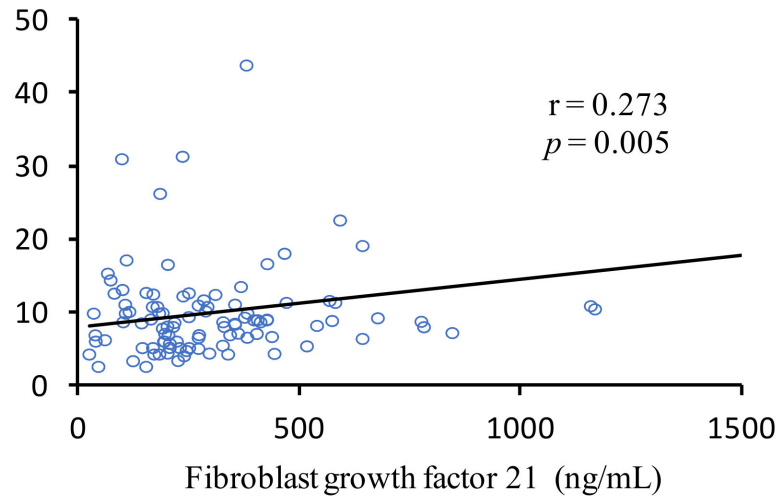

Figure 2. Relationships of the levels of fibroblast growth factor 19 with the levels of c-peptide (A) and $\mathrm{HbA1c}(\mathbf{B})$, and fibroblast growth factor 19 with c-peptide (C) and total bile acids (D), in patients with obesity and type 2 diabetes mellitus at M0, M3, and M12.

\subsection{Characteristic Differences between DM-CR and DM-Non-CR Subjects}

Thirteen of our 35 study participants (37.1\%) achieved complete DM remission 12 months after GB. Profiles of both DM-CR and DM-non-CR groups measured before and 12 months after GB are presented in Table 2.

Regarding the preoperative condition, the DM-CR group had higher baseline body weight (93.85 \pm 16.25 vs. $79.43 \pm 9.53 \mathrm{~kg}, p=0.010)$, BMI $\left(43.73 \pm 5.00\right.$ vs. $29.79 \pm 3.27 \mathrm{~kg} / \mathrm{m}^{2}$, $p=0.001)$, waist circumference $(108.69 \pm 10.22$ vs. $100.45 \pm 9.13 \mathrm{~cm}, p=0.020)$, cpeptide ( $3.23 \pm 1.01$ vs. $2.31 \pm 1.18 \mathrm{mg} / \mathrm{dL}, p=0.026)$, and ALT level $(56.08 \pm 40.45 \mathrm{vs}$. $33.05 \pm 25.90 \mathrm{U} / \mathrm{L}, p=0.047)$ but with lower baseline HbA1c (8.51 \pm 1.42 vs. $9.76 \pm 1.41 \%$, $p=0.016)$ compared to the DM-non-CR group. In addition, the DM-CR group had higher HSI (51.30 \pm 5.05 vs. $42.69 \pm 4.75, p<0.001)$. Baseline FGF 19 , FGF 21 , and total bile acid levels were similar between the two groups.

One year after the operation, the HbA1c level of the DM-CR group was $5.42 \pm 0.38 \%$, while that of the DM-non-CR group was $7.09 \pm 0.99 \%$. Patients who achieved DM-CR had lower fasting blood glucose $(89.70 \pm 10.22$ vs. $125.18 \pm 31.18 \mathrm{mg} / \mathrm{dL}, p<0.001)$, $\gamma$-GT $(12.40 \pm 5.30$ vs. $31.45 \pm 24.71 \mathrm{U} / \mathrm{L}, p=0.003)$, and triglyceride $(74.00 \pm 24.10 \mathrm{vs}$. $119.45 \pm 46.79 \mathrm{mg} / \mathrm{dL}, p=0.001$ ) levels. No significant difference was found among other measurements regarding metabolic profiles or laboratory data. Neither FGF 19, FGF 21, nor total bile acid level showed any significant difference between DM-CR and DM-non-CR 12 months after GB. 
Table 2. Characteristics of obese patients with type 2 diabetes mellitus before and 1 year after gastric bypass between the complete remitters of diabetes mellitus (DM-CR) and non-complete remitters of diabetes mellitus (DM-non-CR).

\begin{tabular}{|c|c|c|c|c|c|c|}
\hline & \multicolumn{3}{|c|}{ M0 } & \multicolumn{3}{|c|}{ M12 } \\
\hline & $\begin{array}{l}\text { DM-CR } \\
(n=13)\end{array}$ & $\begin{array}{l}\text { DM-Non-CR } \\
\quad(n=22)\end{array}$ & $\begin{array}{c}p \\
\text { Value }\end{array}$ & $\begin{array}{l}\text { DM-CR } \\
(n=13)\end{array}$ & $\begin{array}{c}\text { DM-Non-CR } \\
\quad(n=22)\end{array}$ & $\begin{array}{c}p \\
\text { Value }\end{array}$ \\
\hline Metabolic profile & & & & & & \\
\hline Body weight (kg) & $93.85 \pm 16.25$ & $79.42 \pm 9.53$ & 0.010 & $62.6 \pm 7.78$ & $64.43 \pm 6.38$ & 0.511 \\
\hline BMI $\left(\mathrm{kg} / \mathrm{m}^{2}\right)$ & $34.73 \pm 5.00$ & $29.79 \pm 3.27$ & 0.001 & $24.88 \pm 4.08$ & $24.20 \pm 1.64$ & 0.641 \\
\hline Waist circumference $(\mathrm{cm})$ & $108.69 \pm 10.22$ & $100.45 \pm 9.13$ & 0.020 & $80.56 \pm 4.61$ & $83.13 \pm 5.51$ & 0.234 \\
\hline ABSI & $0.080 \pm 0.006$ & $0.082 \pm 0.004$ & 0.246 & $0.076 \pm 0.006$ & $0.078 \pm 0.003$ & 0.584 \\
\hline Systolic blood pressure (mmHg) & $130.92 \pm 13.45$ & $139.14 \pm 15.07$ & 0.115 & $124.00 \pm 20.70$ & $128.00 \pm 14.91$ & 0.624 \\
\hline $\begin{array}{l}\text { Diastolic blood pressure (mmHg) } \\
\text { Laboratory data }\end{array}$ & $83.54 \pm 10.82$ & $86.73 \pm 10.75$ & 0.404 & $75.33 \pm 14.07$ & $81.0 \pm 14.29$ & 0.389 \\
\hline Creatinine $(\mathrm{mg} / \mathrm{dL})$ & $0.67 \pm 0.15$ & $0.86 \pm 0.37$ & 0.051 & $0.60 \pm 0.11$ & $0.76 \pm 0.33$ & 0.063 \\
\hline Fasting blood glucose (mg/dL) & $164.69 \pm 64.95$ & $183.73 \pm 74.34$ & 0.449 & $89.70 \pm 10.22$ & $125.18 \pm 31.18$ & $<0.001$ \\
\hline $\operatorname{HbA} 1 \mathrm{c}(\%)$ & $8.51 \pm 1.42$ & $9.76 \pm 1.41$ & 0.016 & $5.42 \pm 0.38$ & $7.09 \pm 0.99$ & $<0.001$ \\
\hline C-peptide (mg/dL) & $3.23 \pm 1.01$ & $2.31 \pm 1.18$ & 0.026 & $1.35 \pm 0.34$ & $1.41 \pm 0.57$ & 0.791 \\
\hline Insulin (mU/L) & $18.05 \pm 9.24$ & $26.19 \pm 35.75$ & 0.321 & $4.99 \pm 3.07$ & $6.24 \pm 8.11$ & 0.645 \\
\hline HOMA-IR index & $7.21 \pm 3.95$ & $11.51 \pm 16.55$ & 0.256 & $1.06 \pm 0.67$ & $1.93 \pm 2.38$ & 0.152 \\
\hline HOMA- $\beta$ index & $0.81 \pm 0.59$ & $1.34 \pm 2.41$ & 0.331 & $0.61 \pm 0.64$ & $0.44 \pm 0.69$ & 0.479 \\
\hline $\operatorname{ALT}(\mathrm{U} / \mathrm{L})$ & $56.08 \pm 40.45$ & $33.05 \pm 25.90$ & 0.047 & $24.40 \pm 17.56$ & $37.52 \pm 38.16$ & 0.200 \\
\hline AST (U/L) & $37.00 \pm 27.61$ & $29.32 \pm 28.61$ & 0.443 & $22.70 \pm 12.05$ & $31.86 \pm 30.52$ & 0.371 \\
\hline Alk-p (U/L) & $64.46 \pm 24.37$ & $59.68 \pm 15.55$ & 0.482 & $62.70 \pm 16.57$ & $73.19 \pm 20.18$ & 0.164 \\
\hline$\gamma-\mathrm{GT}(\mathrm{U} / \mathrm{L})$ & $51.58 \pm 34.04$ & $36.42 \pm 22.19$ & 0.143 & $12.40 \pm 5.30$ & $31.45 \pm 24.71$ & 0.003 \\
\hline Total cholesterol (mg/dL) & $186.62 \pm 49.00$ & $197.73 \pm 39.54$ & 0.468 & $155.50 \pm 24.65$ & $173.82 \pm 35.50$ & 0.151 \\
\hline Triglyceride (mg/dL) & $179.08 \pm 133.37$ & $262.27 \pm 252.47$ & 0.212 & $74.00 \pm 24.10$ & $119.45 \pm 46.79$ & 0.001 \\
\hline HDL-C (mg/dL) & $39.85 \pm 7.71$ & $42.59 \pm 7.85$ & 0.322 & $45.80 \pm 7.94$ & $46.55 \pm 11.28$ & 0.852 \\
\hline LDL-C (mg/dL) & $119.46 \pm 39.61$ & $116.95 \pm 31.00$ & 0.836 & $95.10 \pm 19.71$ & $110.50 \pm 33.34$ & 0.187 \\
\hline Uric acid (mg/dL) & $5.69 \pm 1.58$ & $5.61 \pm 1.69$ & 0.886 & $4.63 \pm 1.18$ & $5.33 \pm 1.61$ & 0.230 \\
\hline Total bile acid $(\mu \mathrm{M})$ & $9.97 \pm 3.63$ & $10.13 \pm 4.77$ & 0.919 & $7.61 \pm 2.91$ & $8.72 \pm 5.86$ & 0.462 \\
\hline FGF $19(\mathrm{pg} / \mathrm{mL})$ & $63.73 \pm 40.17$ & $96.29 \pm 68.93$ & 0.131 & $162.41 \pm 131.83$ & $131.03 \pm 77.10$ & 0.445 \\
\hline FGF $21(\mathrm{pg} / \mathrm{mL})$ & $370.14 \pm 300.53$ & $290.47 \pm 195.89$ & 0.348 & $221.17 \pm 93.30$ & $235.60 \pm 140.44$ & 0.744 \\
\hline HSI & $51.30 \pm 5.05$ & $42.69 \pm 4.75$ & $<0.001$ & $36.08 \pm 3.10$ & $36.33 \pm 2.44$ & 0.831 \\
\hline
\end{tabular}

ABSI, a body shape index; BMI, body mass index; HbA1c, hemoglobin A1c; HOMA, homeostasis model assessment; IR, insulin resistance; ALT, alanine aminotransferase; AST, aspartate transaminase; Alk-p, alkaline phosphatase; $\gamma$-GT, gamma-glutamyl transferase; HDL-C, high-density lipoprotein cholesterol; LDL-C, lowdensity lipoprotein cholesterol; FGF, fibroblast growth factor; HSI, hepatic steatosis index.

Changes in serum levels of FGF 19, FGF 21, and total bile acids at the time before operation and 3 months and 12 months after surgery are presented in Figure 3. After adjustment for age and gender, the DM-CR group had a significantly higher FGF 19 level compared to DM-non-CR at M3 (196.88 \pm 153.00 vs. $102.06 \pm 34.72 \mathrm{pg} / \mathrm{mL}, p=0.004$, shown in Figure 3B), and more changes in FGF 19 between M3 and M0 (133.15 \pm 144.65 vs. $6.15 \pm 86.35 \mathrm{pg} / \mathrm{mL}, p=0.001$, shown in Figure 3D) compared with the DM-non-CR group. 

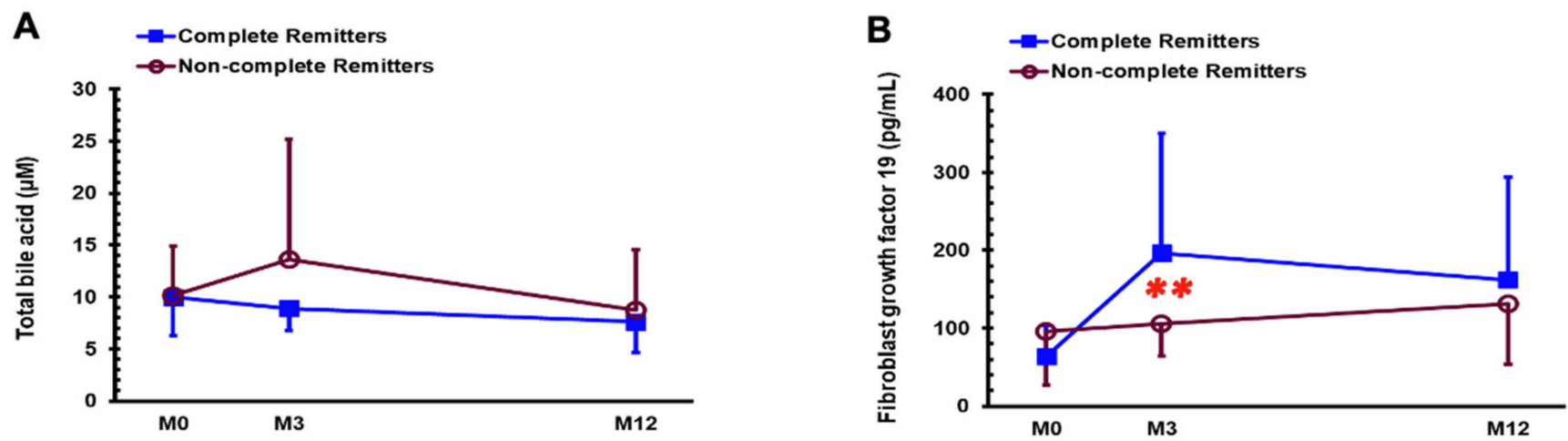

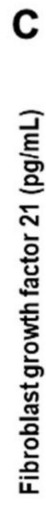
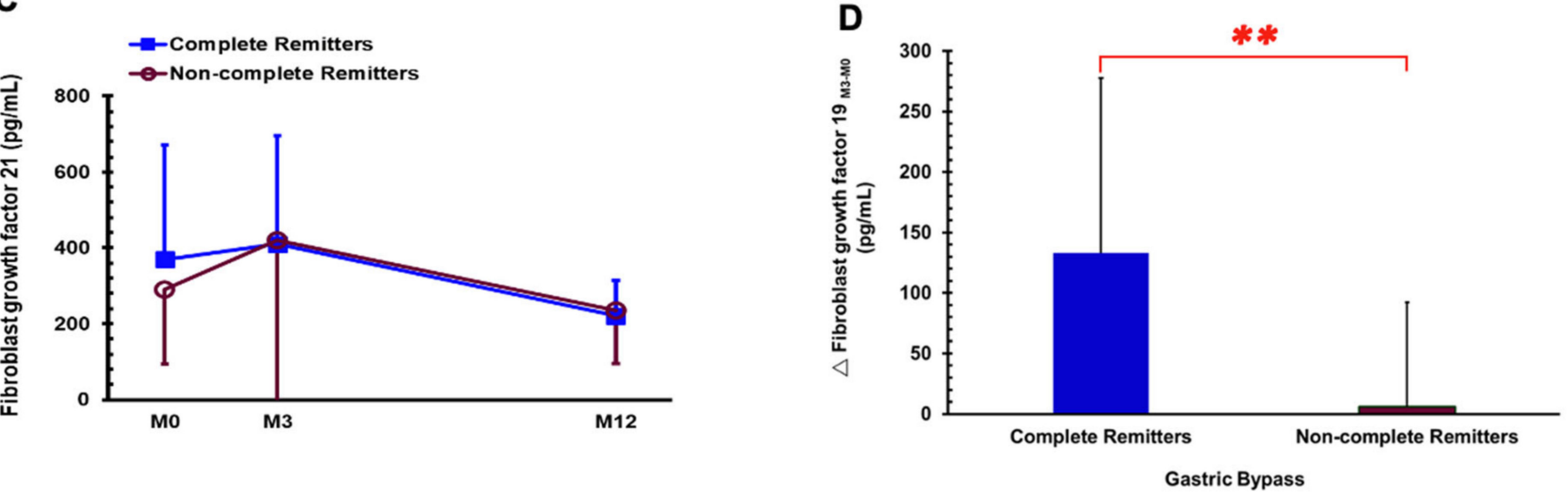

Figure 3. Serum levels of total bile acids (A), fibroblast growth factor 19 (B), fibroblast growth factor 21 (C) in the DM-CR and DM-non-CR groups before gastric bypass (M0) and 3 months (M3) and 1 year (M12) postoperatively and change in fibroblast growth factor 19 at 3 months after gastric bypass (D). ${ }^{* *} p<0.01$ compared between the DM-CR and DM-non-CR groups after adjusted for age and gender.

\subsection{Characteristic Differences between HSI-I and HSI-Non-I Subjects}

Twenty-five of our 35 enrolled subjects (71.4\%) were considered HSI-I based on evaluation at M12. Measurements of HSI-I and HSI-non-I groups before and at 12 months postoperatively are presented in Table 3.

Regarding the preoperative condition, patients among the HSI-I group had higher systolic blood pressure (139.60 \pm 15.11 vs. $127.30 \pm 10.14 \mathrm{mmHg}, p=0.024)$ and lower fasting blood glucose (161.44 \pm 66.11 vs. $214.70 \pm 70.31, p=0.042)$. Neither baseline FGF 19, FGF 21, nor total bile acid level showed any significant difference between these two groups.

One year after GB, fatty liver improvers had an average HSI of $34.49 \pm 1.25$, while non-improvers had a HSI of $38.70 \pm 1.93$. HSI-I had lower serum FGF $21(204.06 \pm 122.68$ vs. $295.67 \pm 104.96, p=0.046)$, insulin $(3.43 \pm 1.78$ vs. $10.88 \pm 10.38 \mathrm{mU} / \mathrm{L}, p=0.0499)$, and triglyceride levels $(90.18 \pm 32.01$ vs. $138.40 \pm 55.67 \mathrm{mg} / \mathrm{dL}, p=0.026)$. No differences in FGF 19 and bile acid levels were found. 
Table 3. Characteristics of obese patients with type 2 diabetes mellitus before and 1 year after gastric bypass between the hepatic steatosis index improvers (HSI-I) and hepatic steatosis index non-improvers (HSI-non-I).

\begin{tabular}{|c|c|c|c|c|c|c|}
\hline & \multicolumn{3}{|c|}{ M0 } & \multicolumn{3}{|c|}{ M12 } \\
\hline & HSI-I $(n=25)$ & $\begin{array}{l}\text { HSI-Non-I } \\
\quad(n=10)\end{array}$ & $\begin{array}{c}p \\
\text { Value }\end{array}$ & HSI-I $(n=25)$ & $\begin{array}{l}\text { HSI-Non-I } \\
\quad(n=10)\end{array}$ & $\begin{array}{c}p \\
\text { Value }\end{array}$ \\
\hline Metabolic profile & & & & & & \\
\hline Body weight $(\mathrm{kg})$ & $86.19 \pm 15.56$ & $81.26 \pm 9.38$ & 0.358 & $62.42 \pm 6.57$ & $66.59 \pm 6.55$ & 0.116 \\
\hline BMI $\left(\mathrm{kg} / \mathrm{m}^{2}\right)$ & $31.98 \pm 5.14$ & $30.73 \pm 2.98$ & 0.476 & $23.96 \pm 2.78$ & $25.27 \pm 2.02$ & 0.199 \\
\hline Waist circumference (cm) & $104.04 \pm 11.09$ & $102.55 \pm 8.31$ & 0.705 & $81.11 \pm 4.30$ & $84.65 \pm 6.43$ & 0.087 \\
\hline ABSI & $0.081 \pm 0.005$ & $0.082 \pm 0.004$ & 0.606 & $0.077 \pm 0.004$ & $0.077 \pm 0.005$ & 0.995 \\
\hline Systolic blood pressure (mmHg) & $139.60 \pm 15.11$ & $127.30 \pm 10.14$ & 0.024 & $129.58 \pm 15.08$ & $123.22 \pm 18.07$ & 0.390 \\
\hline $\begin{array}{c}\text { Diastolic blood pressure }(\mathrm{mmHg}) \\
\text { Laboratory data }\end{array}$ & $87.00 \pm 11.60$ & $81.90 \pm 7.46$ & 0.209 & $80.92 \pm 14.49$ & $78.00 \pm 14.37$ & 0.652 \\
\hline Creatinine $(\mathrm{mg} / \mathrm{dL})$ & $0.82 \pm 0.36$ & $0.72 \pm 0.13$ & 0.438 & $0.72 \pm 0.35$ & $0.68 \pm 0.070$ & 0.542 \\
\hline Fasting blood glucose (mg/dL) & $161.44 \pm 66.11$ & $214.70 \pm 70.31$ & 0.042 & $108.91 \pm 25.25$ & $125.50 \pm 40.45$ & 0.166 \\
\hline $\operatorname{HbA} 1 \mathrm{c}(\%)$ & $9.11 \pm 1.70$ & $9.76 \pm 0.83$ & 0.258 & $6.41 \pm 1.11$ & $6.72 \pm 1.30$ & 0.482 \\
\hline C-peptide (mg/dL) & $2.51 \pm 1.17$ & $3.02 \pm 1.22$ & 0.255 & $1.27 \pm 0.50$ & $1.63 \pm 0.44$ & 0.062 \\
\hline Insulin $(\mathrm{mU} / \mathrm{L})$ & $21.70 \pm 24.92$ & $26.82 \pm 38.45$ & 0.643 & $3.43 \pm 1.78$ & $10.88 \pm 10.38$ & 0.050 \\
\hline HOMA-IR index & $7.94 \pm 7.82$ & $14.83 \pm 21.82$ & 0.353 & $0.81 \pm 0.49$ & $3.16 \pm 2.80$ & 0.027 \\
\hline HOMA- $\beta$ index & $1.33 \pm 2.23$ & $0.70 \pm 0.88$ & 0.397 & $0.33 \pm 0.38$ & $0.93 \pm 1.01$ & 0.098 \\
\hline $\operatorname{ALT}(\mathrm{U} / \mathrm{L})$ & $37.32 \pm 32.73$ & $52.30 \pm 34.57$ & 0.237 & $35.81 \pm 39.21$ & $28.00 \pm 14.54$ & 0.428 \\
\hline AST (U/L) & $27.36 \pm 21.08$ & $44.20 \pm 39.62$ & 0.110 & $32.00 \pm 33.78$ & $22.40 \pm 10.20$ & 0.209 \\
\hline Alk-p (U/L) & $64.20 \pm 20.51$ & $54.60 \pm 13.49$ & 0.183 & $70.33 \pm 20.21$ & $68.70 \pm 18.79$ & 0.831 \\
\hline$\gamma-\mathrm{GT}(\mathrm{U} / \mathrm{L})$ & $43.32 \pm 31.22$ & $39.78 \pm 18.53$ & 0.754 & $24.75 \pm 19.72$ & $25.80 \pm 27.64$ & 0.905 \\
\hline Total cholesterol (mg/dL) & $193.40 \pm 40.09$ & $194.10 \pm 51.72$ & 0.966 & $168.18 \pm 34.42$ & $167.90 \pm 32.25$ & 0.983 \\
\hline Triglyceride (mg/dL) & $189.52 \pm 115.25$ & $336.00 \pm 355.37$ & 0.231 & $90.18 \pm 32.01$ & $138.40 \pm 55.67$ & 0.026 \\
\hline HDL-C (mg/dL) & $41.92 \pm 7.04$ & $40.70 \pm 9.83$ & 0.682 & $47.82 \pm 9.13$ & $43.00 \pm 12.18$ & 0.223 \\
\hline LDL-C (mg/dL) & $122.44 \pm 29.39$ & $106.50 \pm 42.84$ & 0.214 & $105.36 \pm 33.32$ & $106.40 \pm 23.94$ & 0.930 \\
\hline Uric acid (mg/dL) & $5.84 \pm 1.57$ & $5.13 \pm 1.75$ & 0.682 & $5.27 \pm 1.67$ & $4.77 \pm 1.08$ & 0.399 \\
\hline Total bile acid $(\mu \mathrm{M})$ & $10.43 \pm 4.30$ & $9.17 \pm 4.50$ & 0.444 & $8.71 \pm 5.38$ & $7.31 \pm 3.71$ & 0.457 \\
\hline FGF $19(\mathrm{pg} / \mathrm{mL})$ & $87.83 \pm 63.63$ & $75.11 \pm 57.23$ & 0.587 & $144.15 \pm 98.45$ & $139.04 \pm 109.87$ & 0.894 \\
\hline FGF $21(\mathrm{pg} / \mathrm{mL})$ & $314.54 \pm 252.60$ & $333.85 \pm 212.77$ & 0.833 & $204.06 \pm 122.68$ & $295.67 \pm 104.96$ & 0.046 \\
\hline HSI & $46.29 \pm 6.80$ & $44.88 \pm 5.42$ & 0.562 & $34.49 \pm 1.25$ & $38.70 \pm 1.93$ & $<0.001$ \\
\hline
\end{tabular}

ABSI, a body shape index; BMI, body mass index; HbA1c, hemoglobin A1c; HOMA, homeostasis model assessment; IR, insulin resistance; ALT, alanine aminotransferase; AST, aspartate transaminase; Alk-p, alkaline phosphatase; $\gamma$-GT, gamma-glutamyl transferase; HDL-C, high-density lipoprotein cholesterol; LDL-C, lowdensity lipoprotein cholesterol; FGF, fibroblast growth factor; HSI, hepatic steatosis index.

The changes in FGF 19, FGF 21, and total bile acids are presented in Figure 4. The differences in FGF 21 between HSI-I and HSI-non-I showed borderline significance $(p=0.0503)$ after being adjusted for age and gender (Figure 4C). 

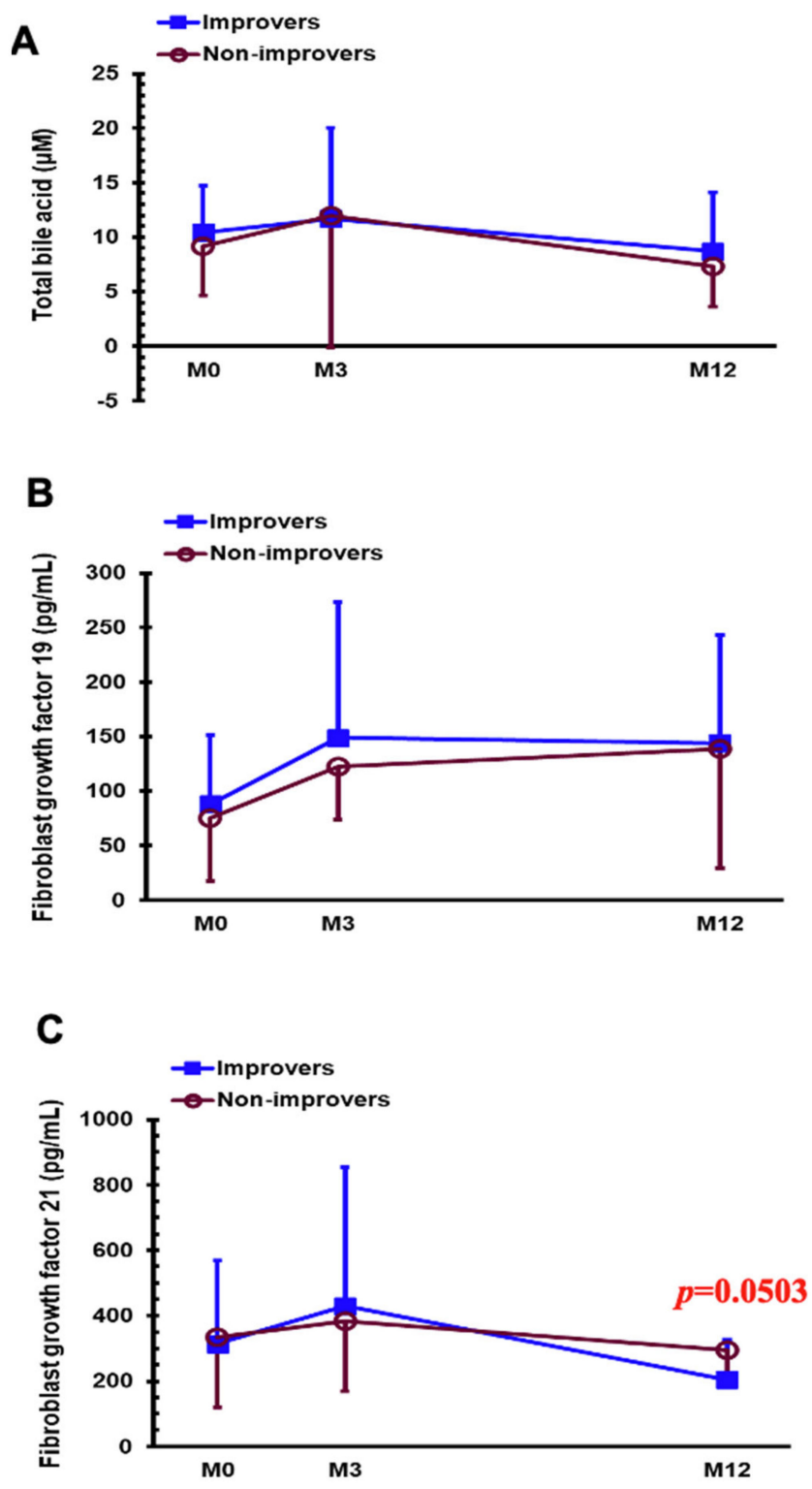

Figure 4. Serum levels of total bile acids (A), fibroblast growth factor 19 (B), and fibroblast growth factor 21 (C) in the HSI-I and HSI-non-I groups before gastric bypass (M0) and 3 months (M3) and 1 year (M12) postoperatively ( $p$ value evaluated after adjusted for age and gender).

\section{Discussion}

In our study, 35 obese patients with T2DM showed significant improvements in body weight, BMI, waist circumference, ABSI and insulin resistance, c-peptide, and insulin after receiving GB. Despite the limited effect in the improvement on lipid profile and liver function, our study also demonstrated GB as a beneficial approach to improving NAFLD based on the significant improvement of HSI after GB. Our study confirmed GB as an effective strategy to combat morbid obesity and its related comorbidities, such as T2DM and NAFLD.

Serum FGF 19 concentrations are consistently elevated in obese patients after GB. This finding is consistent with previous studies in patients receiving sleeve gastrectomy, another standard bariatric procedure [8,47]. FGF 19 has a close connection with obesity $[47,48]$ and correlates negatively with BMI in obese patients with DM [22]. A recent meta-analysis demonstrated a negative association between FGF 19 levels and the degree of BMI reduction after bariatric surgery [49], while obesity and DM led to significantly lower FGF 19 levels compared to those without DM [48]. A recent study from Gómez-Ambrosi et al. [33] 
showed a subsequent increase in serum FGF 19 levels after either diet- or surgery-induced weight loss.

Another major purpose of our study was to provide additional information on the characteristics of FGF levels on DM remission. Our analysis showed a significant elevation of FGF 19 levels among DM remitters compared with non-remitters 3 months after GB. Early FGF 19 improvements may predict the complete remission of T2DM for obese patients receiving GB. Furthermore, our study pointed out a negative linear correlation between FGF 19 levels and the indicators of DM severity, including HbA1c and c-peptide levels. This implies that FGF 19 may also provide predictive value regarding improvements in insulin resistance and remission of T2DM.

FGF 21 signaling plays a crucial role in the development and progression of NAFLD [50]. Based on animal studies, the overexpression of FGF 21 antagonizes the effect of FGF 15/19 [51]. The elevation of FGF 21 levels in NAFLD patients may result from dysfunctional PPAR $\alpha$ signaling [52]. Similar to the presence of insulin resistance in T2DM, "FGF 21 resistance" has been proposed as a key feature in NAFLD [53].

In one human study, FGF 21 had a positive correlation with BMI, and the expression of hepatic FGF 21 mRNA was significantly elevated in NAFLD [30]. It is an interesting fact, however, that the presence of FGF 21 elevation only presented during the stage of simple steatosis, and not after the development of non-alcoholic steatohepatitis or the resolution of steatosis [54]. This may be explained as reflecting the anti-inflammation effect of FGF 21 [55].

Our investigation showed that NAFLD improvers tended to have lower FGF 21 levels at 12 months after GB with borderline significance. This finding consisted of the effect of bariatric surgery on reducing hepatic fat, inflammation, and fibrosis [56]. Recent studies pointed out potential differences from the aspect of different surgical interventions [33,57]. The effect on changes in FGF 21 is more prominent in sleeve gastrectomy compared with GB [33]. Our previous instigation also showed a more prominent change in pancreatic polypeptide hormone after receiving sleeve gastrectomy [57]. The results from our current study agree with this finding and further comparison between different types of surgery with larger study populations may be required.

Despite FGF 19 and FGF 21 displaying some overlapping functions, our study showed differential roles of FGF 19 and FGF 21. FGF 19 had a higher affinity toward FGFr4, while FGF 21 is more potent toward FGFr1c. The FGFr4 gene is mainly expressed in the liver, whereas the FGFr1c gene has higher expression in the adipose tissue [58]. The biological evidence supports the fact that FGF 19 tends to have a closer interaction with DM and insulin resistance, while FGF 21 plays a greater role in the spectrum of fatty liver disease. Our study supports the notion that both FGF 19 and 21 may have complementary advantages in evaluating these two major comorbidities, T2DM and NAFLD, of obesity, respectively.

The understanding of these energy-regulating pathways suggests the potential of pharmacological approaches for patients with T2DM and NAFLD. Aldafermin (NGM 282), an engineered FGF 19 analog, demonstrated its safety and effectiveness in reducing liver fat content, improving liver fibrosis, and preventing the progression of non-alcoholic steatohepatitis (NASH) in a recent phase 2 randomized control trial [59], whereas its ability to improve insulin sensitivity remained inconsistent among studies [60]. Pegbelfermin and efruxifermin are considered the two most promising FGF 21 analogs. In a phase 2 study focusing on morbidly obese T2DM patients, high-dose pegbelfermin (BMS-986036), a PEGylated form of FGF 21, showed an effect on improving the lipid profile (increasing HDL-C and lower triglycerides), while it had no significant effect on improving weight loss or glycemic control [61]. Efruxifermin, a fusion protein of human IgG1 FC domain linked to a modified human FGF 21, is considered a promising agent in reducing the liver fat fraction and markers of hepatic injury among NASH patients, as reported in a recent phase 2a study [62]. There is still limited evidence focusing on the obese population or on the aspect of combination or comparison with bariatric surgery. 
Our study has several limitations. First, the sample size of our study is small and may neglect the potential differences in the two biomarkers while performing subgroup analysis. Second, the current diagnostic criteria of NAFLD depend on histological or image studies. Subjects in our study did not receive evaluations of NAFLD during enrollment, and we adapted HSI as a blood surrogate for non-invasive assessment. The HSI has been validated with other non-invasive approaches, including ultrasonographic and magnetic resonance imaging [46]. HSI is also widely adopted in the field of metabolic disorders [63,64].

\section{Conclusions}

Obesity represents a major health challenge in our modern society. GB is an effective surgical approach for weight loss, leading to an increase in FGF 19 levels and a decrease in total bile acids and FGF 21 levels postoperatively. FGF 19 levels had a negative correlation with the severity of T2DM based on c-peptide and HbA1c levels. FGF 21 levels had a positive correlation with c-peptide and total bile acid levels. Early increases in serum FGF 19 levels may be a predictor for complete remission of T2DM after GB. A decline in serum FGF 21 levels may reflect the improvement of NAFLD after GB. Our study may shed light on the differential roles of FGF 19 and FGF 21 in human T2DM remission and NAFLD improvement.

Author Contributions: Conceptualization, J.-Y.G. and C.-Y.C.; methodology, J.-Y.G., H.-H.C., W.-J.L. and C.-Y.C.; software, H.-H.C.; validation, W.-J.L., S.-C.C. and C.-Y.C.; formal analysis, H.-H.C.; investigation, J.-Y.G. and W.-J.L.; resources, W.-J.L.; data curation, H.-H.C.; writing-original draft preparation, J.-Y.G.; writing-review and editing, J.-Y.G. and S.-C.C.; visualization, H.-H.C.; supervision, W.-J.L., S.-C.C., S.-D.L. and C.-Y.C.; project administration, C.-Y.C.; funding acquisition, W.-J.L. and C.-Y.C. All authors have read and agreed to the published version of the manuscript.

Funding: This research was funded by grants from Min-Sheng General Hospital, Taoyuan, the Taiwan Ministry of Science and Technology (MOST 104-2314-B-010-005-) and intramural grants from Taipei Veterans General Hospital, Taipei, Taiwan (V99F-010 and V101C-118), Cheng Hsin General Hospital, and the National Yang-Ming University Joint Research Program (103F003C13).

Institutional Review Board Statement: The study was conducted according to the guidelines of the Declaration of Helsinki and approved by the institutional review board (approval number: MSIRB2015017, YM 103127E, 201002037IC, 2011-09-015IC, CHGH-IRB (405)102A-52)).

Informed Consent Statement: Informed consent was obtained from all subjects involved in the study.

Data Availability Statement: The dataset are available from the corresponding author at chency@vghtpe.gov.tw.

Acknowledgments: We thank all participants who were willing to attend this investigation.

Conflicts of Interest: The authors declare no conflict of interest.

\section{References}

1. Blüher, M. Obesity: Global epidemiology and pathogenesis. Nat. Rev. Endocrinol. 2019, 15, 288-298. [CrossRef]

2. Chiang, D.J.; Pritchard, M.T.; Nagy, L.E. Obesity, diabetes mellitus, and liver fibrosis. Am. J. Physiol.-Gastrointest. Liver Physiol. 2011, 300, G697-G702. [CrossRef] [PubMed]

3. Clamp, L.D.; Hume, D.J.; Lambert, E.V.; Kroff, J. Enhanced insulin sensitivity in successful, long-term weight loss maintainers compared with matched controls with no weight loss history. Nutr. Diabetes 2017, 7, e282. [CrossRef] [PubMed]

4. Yoshioka, N.; Ishigami, M.; Watanabe, Y.; Sumi, H.; Doisaki, M.; Yamaguchi, T.; Ito, T.; Ishizu, Y.; Kuzuya, T.; Honda, T.; et al. Effect of weight change and lifestyle modifications on the development or remission of nonalcoholic fatty liver disease: Sex-specific analysis. Sci. Rep. 2020, 10, 481. [CrossRef]

5. Di Lorenzo, N.; Antoniou, S.A.; Batterham, R.L.; Busetto, L.; Godoroja, D.; Iossa, A.; Carrano, F.M.; Agresta, F.; Alarçon, I.; Azran, C.; et al. Clinical practice guidelines of the European Association for Endoscopic Surgery (EAES) on bariatric surgery: Update 2020 endorsed by IFSO-EC, EASO and ESPCOP. Surg. Endosc. 2020, 34, 2332-2358. [CrossRef] [PubMed]

6. Schauer, P.R.; Bhatt, D.L.; Kirwan, J.P.; Wolski, K.; Brethauer, S.A.; Navaneethan, S.D.; Aminian, A.; Pothier, C.E.; Kim, E.S.; Nissen, S.E.; et al. Bariatric surgery versus intensive medical therapy for diabetes-3-year outcomes. N. Engl. J. Med. 2014, 370, 2002-2013. [CrossRef] [PubMed] 
7. Jurowich, C.; Thalheimer, A.; Hartmann, D.; Bender, G.; Seyfried, F.; Germer, C.T.; Wichelmann, C. Improvement of type 2 diabetes mellitus (T2DM) after bariatric surgery-who fails in the early postoperative course? Obes. Surg. 2012, 22, 1521-1526. [CrossRef]

8. Huang, H.H.; Lee, W.J.; Chen, S.C.; Chen, T.F.; Lee, S.D.; Chen, C.Y. Bile Acid and Fibroblast Growth Factor 19 Regulation in Obese Diabetics, and Non-Alcoholic Fatty Liver Disease after Sleeve Gastrectomy. J. Clin. Med. 2019, 8, 815. [CrossRef]

9. Bastos, E.C.; Barbosa, E.M.; Soriano, G.M.; dos Santos, E.A.; Vasconcelos, S.M. Determinants of weight regain after bariatric surgery. Arq. Bras. De Cir. Dig. 2013, 26, 26-32. [CrossRef]

10. Wu, W.C.; Lee, W.J.; Lee, T.H.; Chen, S.C.; Chen, C.Y. Do different bariatric surgical procedures influence plasma levels of matrix metalloproteinase-2, -7, and -9 among patients with type 2 diabetes mellitus? World J. Diabetes 2020, 11, 252-260. [CrossRef]

11. Júnior, W.S.; Nonino-Borges, C.B. Clinical predictors of different grades of nonalcoholic fatty liver disease. Obes. Surg. 2012, 22, 248-252. [CrossRef] [PubMed]

12. Guo, J.Y.; Chen, J.C.; Chen, C.Y. Is there any useful surrogate to evaluate metabolic fatty liver disease? J. Chin. Med. Assoc. 2021, 84, 344-345. [CrossRef] [PubMed]

13. Owen, B.M.; Mangelsdorf, D.J.; Kliewer, S.A. Tissue-specific actions of the metabolic hormones FGF15/19 and FGF21. Trends Endocrinol. Metab. 2015, 26, 22-29. [CrossRef] [PubMed]

14. Fu, T.; Kemper, J.K. MicroRNA-34a and Impaired FGF19/21 Signaling in Obesity. Vitam. Horm. 2016, 101, 175-196. [CrossRef]

15. Fu, L.; John, L.M.; Adams, S.H.; Yu, X.X.; Tomlinson, E.; Renz, M.; Williams, P.M.; Soriano, R.; Corpuz, R.; Moffat, B.; et al. Fibroblast growth factor 19 increases metabolic rate and reverses dietary and leptin-deficient diabetes. Endocrinology 2004, 145, 2594-2603. [CrossRef]

16. Coskun, T.; Bina, H.A.; Schneider, M.A.; Dunbar, J.D.; Hu, C.C.; Chen, Y.; Moller, D.E.; Kharitonenkov, A. Fibroblast growth factor 21 corrects obesity in mice. Endocrinology 2008, 149, 6018-6027. [CrossRef]

17. Adams, A.C.; Coskun, T.; Rovira, A.R.; Schneider, M.A.; Raches, D.W.; Micanovic, R.; Bina, H.A.; Dunbar, J.D.; Kharitonenkov, A. Fundamentals of FGF19 \& FGF21 action in vitro and in vivo. PLoS ONE 2012, 7, e38438. [CrossRef]

18. Tomlinson, E.; Fu, L.; John, L.; Hultgren, B.; Huang, X.; Renz, M.; Stephan, J.P.; Tsai, S.P.; Powell-Braxton, L.; French, D.; et al. Transgenic mice expressing human fibroblast growth factor-19 display increased metabolic rate and decreased adiposity. Endocrinology 2002, 143, 1741-1747. [CrossRef]

19. Inagaki, T.; Choi, M.; Moschetta, A.; Peng, L.; Cummins, C.L.; McDonald, J.G.; Luo, G.; Jones, S.A.; Goodwin, B.; Richardson, J.A.; et al. Fibroblast growth factor 15 functions as an enterohepatic signal to regulate bile acid homeostasis. Cell Metab. 2005, 2, 217-225. [CrossRef]

20. Katoh, M. FGFR inhibitors: Effects on cancer cells, tumor microenvironment and whole-body homeostasis (Review). Int. J. Mol. Med. 2016, 38, 3-15. [CrossRef]

21. Lundåsen, T.; Gälman, C.; Angelin, B.; Rudling, M. Circulating intestinal fibroblast growth factor 19 has a pronounced diurnal variation and modulates hepatic bile acid synthesis in man. J. Intern. Med. 2006, 260, 530-536. [CrossRef]

22. Barutcuoglu, B.; Basol, G.; Cakir, Y.; Cetinkalp, S.; Parildar, Z.; Kabaroglu, C.; Ozmen, D.; Mutaf, I.; Bayindir, O. Fibroblast growth factor-19 levels in type 2 diabetic patients with metabolic syndrome. Ann. Clin. Lab. Sci. 2011, 41, 390-396. [PubMed]

23. Fon Tacer, K.; Bookout, A.L.; Ding, X.; Kurosu, H.; John, G.B.; Wang, L.; Goetz, R.; Mohammadi, M.; Kuro-o, M.; Mangelsdorf, D.J.; et al. Research resource: Comprehensive expression atlas of the fibroblast growth factor system in adult mouse. Mol. Endocrinol. 2010, 24, 2050-2064. [CrossRef] [PubMed]

24. Markan, K.R.; Naber, M.C.; Ameka, M.K.; Anderegg, M.D.; Mangelsdorf, D.J.; Kliewer, S.A.; Mohammadi, M.; Potthoff, M.J Circulating FGF21 is liver derived and enhances glucose uptake during refeeding and overfeeding. Diabetes 2014, 63, 4057-4063. [CrossRef] [PubMed]

25. Martínez-Garza, Ú.; Torres-Oteros, D.; Yarritu-Gallego, A.; Marrero, P.F.; Haro, D.; Relat, J. Fibroblast Growth Factor 21 and the Adaptive Response to Nutritional Challenges. Int. J. Mol. Sci. 2019, 20, 4692. [CrossRef] [PubMed]

26. Inagaki, T.; Dutchak, P.; Zhao, G.; Ding, X.; Gautron, L.; Parameswara, V.; Li, Y.; Goetz, R.; Mohammadi, M.; Esser, V.; et al. Endocrine regulation of the fasting response by PPARalpha-mediated induction of fibroblast growth factor 21. Cell Metab. 2007, 5, 415-425. [CrossRef]

27. Mai, K.; Andres, J.; Biedasek, K.; Weicht, J.; Bobbert, T.; Sabath, M.; Meinus, S.; Reinecke, F.; Möhlig, M.; Weickert, M.O.; et al. Free fatty acids link metabolism and regulation of the insulin-sensitizing fibroblast growth factor-21. Diabetes 2009, 58, 1532-1538. [CrossRef]

28. Iroz, A.; Montagner, A.; Benhamed, F.; Levavasseur, F.; Polizzi, A.; Anthony, E.; Régnier, M.; Fouché, E.; Lukowicz, C.; Cauzac, M.; et al. A Specific ChREBP and PPAR $\alpha$ Cross-Talk Is Required for the Glucose-Mediated FGF21 Response. Cell Rep. 2017, 21, 403-416. [CrossRef]

29. Laeger, T.; Henagan, T.M.; Albarado, D.C.; Redman, L.M.; Bray, G.A.; Noland, R.C.; Münzberg, H.; Hutson, S.M.; Gettys, T.W.; Schwartz, M.W.; et al. FGF21 is an endocrine signal of protein restriction. J. Clin. Investig. 2014, 124, 3913-3922. [CrossRef]

30. Dushay, J.; Chui, P.C.; Gopalakrishnan, G.S.; Varela-Rey, M.; Crawley, M.; Fisher, F.M.; Badman, M.K.; Martinez-Chantar, M.L.; Maratos-Flier, E. Increased fibroblast growth factor 21 in obesity and nonalcoholic fatty liver disease. Gastroenterology 2010, 139, 456-463. [CrossRef]

31. Tezze, C.; Romanello, V.; Sandri, M. FGF21 as Modulator of Metabolism in Health and Disease. Front. Physiol. 2019, 10, 419. [CrossRef] [PubMed] 
32. Inagaki, T.; Lin, V.Y.; Goetz, R.; Mohammadi, M.; Mangelsdorf, D.J.; Kliewer, S.A. Inhibition of growth hormone signaling by the fasting-induced hormone FGF21. Cell Metab. 2008, 8, 77-83. [CrossRef] [PubMed]

33. Gómez-Ambrosi, J.; Gallego-Escuredo, J.M.; Catalán, V.; Rodríguez, A.; Domingo, P.; Moncada, R.; Valentí, V.; Salvador, J.; Giralt, M.; Villarroya, F.; et al. FGF19 and FGF21 serum concentrations in human obesity and type 2 diabetes behave differently after diet- or surgically-induced weight loss. Clin. Nutr. 2017, 36, 861-868. [CrossRef]

34. Yeh, C.; Huang, H.H.; Chen, S.C.; Chen, T.F.; Ser, K.H.; Chen, C.Y. Comparison of consumption behavior and appetite sensations among patients with type 2 diabetes mellitus after bariatric surgery. Peer] 2017, 5, e3090. [CrossRef]

35. Lee, W.J.; Chen, C.Y.; Chong, K.; Lee, Y.C.; Chen, S.C.; Lee, S.D. Changes in postprandial gut hormones after metabolic surgery: A comparison of gastric bypass and sleeve gastrectomy. Surg. Obes. Relat. Dis. 2011, 7, 683-690. [CrossRef] [PubMed]

36. Lee, W.J.; Chong, K.; Ser, K.H.; Lee, Y.C.; Chen, S.C.; Chen, J.C.; Tsai, M.H.; Chuang, L.M. Gastric bypass vs sleeve gastrectomy for type 2 diabetes mellitus: A randomized controlled trial. Arch. Surg. 2011, 146, 143-148. [CrossRef]

37. Wittgrove, A.C.; Clark, G.W. Laparoscopic gastric bypass, Roux-en-Y- 500 patients: Technique and results, with 3-60 month follow-up. Obes. Surg. 2000, 10, 233-239. [CrossRef]

38. Chiu, C.C.; Lee, W.J.; Wang, W.; Wei, P.L.; Huang, M.T. Prevention of trocar-wound hernia in laparoscopic bariatric operations. Obes. Surg. 2006, 16, 913-918. [CrossRef]

39. Captieux, M.; Prigge, R.; Wild, S.; Guthrie, B. Defining remission of type 2 diabetes in research studies: A systematic scoping review. PLoS Med. 2020, 17, e1003396. [CrossRef]

40. Wallace, T.M.; Levy, J.C.; Matthews, D.R. Use and abuse of HOMA modeling. Diabetes Care 2004, 27, 1487-1495. [CrossRef]

41. Wang, J.W.; Chen, P.Y.; Huang, H.H.; Yeh, C.; Chen, S.C.; Lee, W.J.; Chen, C.Y. Change of plasma amylin after bariatric surgery challenged by oral glucose is associated with remission of type 2 diabetes mellitus. J. Chin. Med. Assoc. 2021, 84, 1001-1006. [CrossRef]

42. Godoy-Matos, A.F.; Silva Júnior, W.S.; Valerio, C.M. NAFLD as a continuum: From obesity to metabolic syndrome and diabetes. Diabetol. Metab. Syndr. 2020, 12, 60. [CrossRef] [PubMed]

43. Tapper, E.B.; Lok, A.S. Use of Liver Imaging and Biopsy in Clinical Practice. N. Engl. J. Med. 2017, 377, 756-768. [CrossRef] [PubMed]

44. Foster, G.R.; Goldin, R.D.; Main, J.; Murray-Lyon, I.; Hargreaves, S.; Thomas, H.C. Management of chronic hepatitis C: Clinical audit of biopsy based management algorithm. BMJ 1997, 315, 453-458. [CrossRef] [PubMed]

45. Jung, T.Y.; Kim, M.S.; Hong, H.P.; Kang, K.A.; Jun, D.W. Comparative Assessment and External Validation of Hepatic Steatosis Formulae in a Community-Based Setting. J. Clin. Med. 2020, 9, 2851. [CrossRef] [PubMed]

46. Lee, J.H.; Kim, D.; Kim, H.J.; Lee, C.H.; Yang, J.I.; Kim, W.; Kim, Y.J.; Yoon, J.H.; Cho, S.H.; Sung, M.W.; et al. Hepatic steatosis index: A simple screening tool reflecting nonalcoholic fatty liver disease. Dig. Liver Dis. 2010, 42, 503-508. [CrossRef] [PubMed]

47. Haluzíková, D.; Lacinová, Z.; Kaválková, P.; Drápalová, J.; Kř́žová, J.; Bártlová, M.; Mráz, M.; Petr, T.; Vítek, L.; Kasalický, M.; et al Laparoscopic sleeve gastrectomy differentially affects serum concentrations of FGF-19 and FGF-21 in morbidly obese subjects. Obesity 2013, 21, 1335-1342. [CrossRef]

48. Gerhard, G.S.; Styer, A.M.; Wood, G.C.; Roesch, S.L.; Petrick, A.T.; Gabrielsen, J.; Strodel, W.E.; Still, C.D.; Argyropoulos, G. A role for fibroblast growth factor 19 and bile acids in diabetes remission after Roux-en-Y gastric bypass. Diabetes Care 2013, 36, 1859-1864. [CrossRef]

49. Ryan, P.M.; Hayward, N.E.; Sless, R.T.; Garwood, P.; Rahmani, J. Effect of bariatric surgery on circulating FGF-19: A systematic review and meta-analysis. Obes. Rev. 2020, 21, e13038. [CrossRef]

50. Tucker, B.; Li, H.; Long, X.; Rye, K.A.; Ong, K.L. Fibroblast growth factor 21 in non-alcoholic fatty liver disease. Metabolism 2019, 101, 153994. [CrossRef]

51. Zhang, J.; Gupte, J.; Gong, Y.; Weiszmann, J.; Zhang, Y.; Lee, K.J.; Richards, W.G.; Li, Y. Chronic Over-expression of Fibroblast Growth Factor 21 Increases Bile Acid Biosynthesis by Opposing FGF15/19 Action. EBioMedicine 2017, 15, 173-183. [CrossRef] [PubMed]

52. Rusli, F.; Deelen, J.; Andriyani, E.; Boekschoten, M.V.; Lute, C.; van den Akker, E.B.; Müller, M.; Beekman, M.; Steegenga, W.T. Fibroblast growth factor 21 reflects liver fat accumulation and dysregulation of signalling pathways in the liver of C57BL/6J mice. Sci. Rep. 2016, 6, 30484. [CrossRef] [PubMed]

53. Fisher, F.M.; Chui, P.C.; Antonellis, P.J.; Bina, H.A.; Kharitonenkov, A.; Flier, J.S.; Maratos-Flier, E. Obesity is a fibroblast growth factor 21 (FGF21)-resistant state. Diabetes 2010, 59, 2781-2789. [CrossRef] [PubMed]

54. Wu, G.; Li, H.; Fang, Q.; Zhang, J.; Zhang, M.; Zhang, L.; Wu, L.; Hou, X.; Lu, J.; Bao, Y.; et al. Complementary Role of Fibroblast Growth Factor 21 and Cytokeratin 18 in Monitoring the Different Stages of Nonalcoholic Fatty Liver Disease. Sci. Rep. 2017, 7, 5095. [CrossRef] [PubMed]

55. Liu, X.; Zhang, P.; Martin, R.C.; Cui, G.; Wang, G.; Tan, Y.; Cai, L.; Lv, G.; Li, Y. Lack of fibroblast growth factor 21 accelerates metabolic liver injury characterized by steatohepatities in mice. Am. J. Cancer Res. 2016, 6, 1011-1025.

56. Laursen, T.L.; Hagemann, C.A.; Wei, C.; Kazankov, K.; Thomsen, K.L.; Knop, F.K.; Grønbæk, H. Bariatric surgery in patients with non-alcoholic fatty liver disease-from pathophysiology to clinical effects. World J. Hepatol. 2019, 11, 138-149. [CrossRef]

57. Park, J.M.; Chiu, C.F.; Chen, S.C.; Lee, W.J.; Chen, C.Y. Changes in post-oral glucose challenge pancreatic polypeptide hormone levels following metabolic surgery: A comparison of gastric bypass and sleeve gastrectomy. Neuropeptides 2020, $81,102032$. [CrossRef] 
58. Chen, M.M.; Hale, C.; Stanislaus, S.; Xu, J.; Véniant, M.M. FGF21 acts as a negative regulator of bile acid synthesis. J. Endocrinol. 2018, 237, 139-152. [CrossRef]

59. Harrison, S.A.; Neff, G.; Guy, C.D.; Bashir, M.R.; Paredes, A.H.; Frias, J.P.; Younes, Z.; Trotter, J.F.; Gunn, N.T.; Moussa, S.E.; et al Efficacy and Safety of Aldafermin, an Engineered FGF19 Analog, in a Randomized, Double-Blind, Placebo-Controlled Trial of Patients With Nonalcoholic Steatohepatitis. Gastroenterology 2021, 160, 219-231. [CrossRef]

60. Henriksson, E.; Andersen, B. FGF19 and FGF21 for the Treatment of NASH-Two Sides of the Same Coin? Differential and Overlapping Effects of FGF19 and FGF21 From Mice to Human. Front. Endocrinol. 2020, 11, 601349. [CrossRef]

61. Charles, E.D.; Neuschwander-Tetri, B.A.; Pablo Frias, J.; Kundu, S.; Luo, Y.; Tirucherai, G.S.; Christian, R. Pegbelfermin (BMS986036), PEGylated FGF21, in Patients with Obesity and Type 2 Diabetes: Results from a Randomized Phase 2 Study. Obesity 2019, 27, 41-49. [CrossRef] [PubMed]

62. Harrison, S.A.; Ruane, P.J.; Freilich, B.L.; Neff, G.; Patil, R.; Behling, C.A.; Hu, C.; Fong, E.; de Temple, B.; Tillman, E.J.; et al. Efruxifermin in non-alcoholic steatohepatitis: A randomized, double-blind, placebo-controlled, phase 2a trial. Nat. Med. 2021, 27, 1262-1271. [CrossRef] [PubMed]

63. Sviklāne, L.; Olmane, E.; Dzērve, Z.; Kupčs, K.; Pīrāgs, V.; Sokolovska, J. Fatty liver index and hepatic steatosis index for prediction of non-alcoholic fatty liver disease in type 1 diabetes. J. Gastroenterol. Hepatol. 2018, 33, 270-276. [CrossRef]

64. Kahl, S.; Straßburger, K.; Nowotny, B.; Livingstone, R.; Klüppelholz, B.; Keßel, K.; Hwang, J.H.; Giani, G.; Hoffmann, B.; Pacini, G.; et al. Comparison of liver fat indices for the diagnosis of hepatic steatosis and insulin resistance. PLoS ONE 2014, 9, e94059. [CrossRef] [PubMed] 